WORKING PAPER · NO. 2021-106

\title{
Indoor Air Quality, Information, and Socio-Economic Status: Evidence from Delhi
}

Michael Greenstone, Kenneth Lee, and Harshil Sahai JANUARY 2021

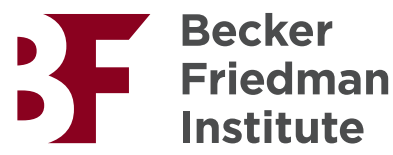


AEA Papers and Proceedings session: Environmental and Energy Economics Around the World Session Chair: Joseph Shapiro

Discussant: Edward Miguel

\title{
Indoor Air Quality, Information, and Socio-Economic Status: Evidence from Delhi
}

\author{
By Michael Greenstone, Kenneth Lee, and Harshil Sahai*
}

Delhi faces some of the world's highest concentrations of $\mathrm{PM}_{2.5}$, the most damaging form of air pollution. Although awareness of outdoor air pollution is rising across the world, there is limited information on indoor air pollution (IAP) levels, particularly in heavily polluted cities like Delhi. Even less evidence exists on how IAP varies by socio-economic status (SES), and whether or not addressing information gaps can change defensive investments against IAP.

In this paper, we deploy Indoor Air Quality Monitors (IAQMs) in thousands of Delhi households across varying socio-economic strata in order to document IAP levels during the peak wintertime air pollution period. Across high and low SES households, we document indoor PM2.5 levels that are: (1) extraordinarily high — more than 20 times World Health Organization (WHO) standards; (2) only 10 percent lower in high (versus low) SES households; and (3) significantly higher than levels reported by the nearest, outdoor government monitors, the main source of public information on air pollution in this setting.

We then report on a field experiment that randomly assigned IAQMs, as well as an opportunity to rent an air purifier at a subsidized price, across medium and high SES homes during

\footnotetext{
* Greenstone: Department of Economics, University of Chicago, 5757 S University Ave, Chicago, IL 60637 (e-mail: mgreenst@uchicago.edu) and NBER; Lee: Department of Economics, University of Chicago, 5757 S University Ave, Chicago, IL 60637 (e-mail: kennethlee@uchicago.edu); Sahai: Department of Economics, University of Chicago, 5757 S University Ave, Chicago, IL 60637 (e-mail: harshil@uchicago.edu). This research was supported by the Tata Centre for Development at UChicago, an initiative of the Tata Trusts in India. We thank Shipra Karan, Rongmon Deka, Tanya Gupta, Shradha Parashari, Ian Pitman, Meghna Singh, and Ramya Teeparthi for superb research assistance, and partners Kaiterra, SmartAir, Indicus Foundation, and Perfect Market Research. All errors are our own.
} 
the 2019-20 winter season. The experiment is limited by significant survey non-response: thousands of medium and high SES Delhi households were approached for recruitment, but only 15 percent were willing, or available, to participate. In addition, 56 percent of households in the treatment group declined the free, month-long user trial with an IAQM, even though the study was carried out during Delhi's peak air pollution period. The sample also suffered from high rates of attrition but is relatively balanced at endline along household characteristics. We find that the IAQM intervention did not lead households to adopt the air purifier rental contract or report any meaningful changes in other defensive investments and actions.

Due to this non-response and attrition, the experiment should be interpreted as suggestive and with caution. However, the basic patterns we observe in our data-including low levels of air purifier ownership, on average; relatively low take-up of a free IAQM user trial; and a lack of interest in the subsidized air purifier rental offers — suggest that in this sample of medium and high SES households, demand for air pollution information and defensive technologies may be low.

\section{Sample and Study Design}

We measure indoor $\mathrm{PM}_{2.5}$ levels using the Kaiterra Laser Egg (KLE), a relatively popular consumer-grade IAQM that retailed for approximately $\$ 135$ during the study period. The KLE monitor is an optical particle counter that measures $\mathrm{PM}_{2.5}$ by drawing air into its sensor and counting the number of particles crossing an internal laser beam. The particle count is calibrated using real-time data from nearby reference grade monitors, which allows for particle counts to be converted into $\mathrm{PM}_{2.5}$. The standard KLE features a back-lit display communicating real-time $\mathrm{PM}_{2.5}$ concentrations in micrograms per cubic meter $\left(\mu \mathrm{g} / \mathrm{m}^{3}\right)$. When connected to a local Wi-Fi network, the KLE transmits minute-wise IAP data to a remote server, accessible to the research team. 


\section{A. Data Collection with Low SES Households}

We utilize data from three sets of households: (1) "low SES" households $(n=3,533)$; (2) "medium and high SES" households ( $n=703)$; and "high SES" households $(n=55) .{ }^{1}$ The "low SES" household sample, which is studied in Lee et al. (2020) and Baylis et al. (2021), is representative of mostly poor, non-migrant individuals living in some of Delhi's poorest neighborhoods. These respondents were surveyed between October 2018 and March 2019 and are drawn from an administrative list of low SES neighborhoods known as the Jhuggie Jhopri Squatter Settlements/Clusters (“J.J. clusters"). At each household, an enumerator administered a survey and manually recorded indoor and outdoor $\mathrm{PM}_{2.5}$ levels, using the KLE monitors described above. Each household was visited up to four times. In total, there are 3,002 households and 6,048 sets of $\mathrm{PM}_{2.5}$ measurements in this sample.

\section{B. Data Collection with Medium and High SES Households}

To identify medium and high SES households, we partnered with Residential Welfare Associations (RWAs) (community groups representing local neighborhoods) across Delhi. We first identified a sample of 49 RWAs, which we subdivided into 90 neighborhoods (or "RWA clusters") prior to recruitment efforts. The RWA clusters were randomly assigned into three groups: (1) "Group A" (j=32), our control group, in which households were surveyed at baseline and endline, roughly one month later; (2) “Group B” (j=28), in which households were also offered a free, month-long trial of a standard KLE monitor, an information intervention on the health impacts of $\mathrm{PM}_{2.5}$, and other pollution-related information; and (3) "Group C" ( $\left.j=30\right)$, in which households were instead offered a modified version of the KLE monitor that lacked a visible display screen, but could still transmit $\mathrm{PM}_{2.5}$ data. Groups A and B households were also provided

\footnotetext{
${ }^{1}$ Appendix A provides details on recruitment, experimental design, sample comparisons, and the full study results.
} 
with opportunities to rent air purifiers (at randomly assigned prices and contract durations) from an international air purifier manufacturer, which could be exercised at any point in the study. Air purifiers are the primary form of defensive technology against IAP in this setting.

We approached 8,877 households for recruitment across Groups A, B, and C, out of which the vast majority ( 85.5 percent) declined or were unable to participate. In Group B, 56.0 percent of households turned down the free user trial with an IAQM. In total, 364 and 339 Group A and Group B households completed the study, respectively (11.0 percent approached for recruitment). The sample further suffered from differential rates of attrition from baseline to endline ( 32 percent and 19 percent for Groups A and B, respectively). In Group C, Wi-Fi connectivity requirements led to further sample restriction: only 55 households successfully paired the modified KLE with local Wi-Fi. This generated nearly a million timestamped, indoor $\mathrm{PM}_{2.5}$ measurements being transmitted to the server. In the remainder of this paper, we refer to the combined Group A and B sample as the "medium and high SES" households, and the Group C sample as the "high SES" households.

Despite significant imbalance at baseline, non-response, and attrition, the sample is relatively balanced at endline, along the household characteristics observed at baseline. We nevertheless interpret experimental results as suggestive and with a high degree of caution.

\section{Sample Characteristics}

In Table A2A, we compare key characteristics between the low SES households (i.e., J.J. clusters), medium and high SES households (i.e., Groups A and B, RWA clusters), and high SES households (i.e., Group C, RWA clusters). Moving from low to high SES: graduated college rises from 11.0 to 39.7 to 65.5 percent; business ownership rises from 4.8 to 13.7 to 16.4 percent; 
number of household members falls from 6.9 to 6.6 to 4.3 ; and capital ownership rises from 10.7 to 53.6 to 89.1 percent for air conditioners, and 1.8 to 4.9 to 24.1 percent for air purifiers.

\section{Patterns of Indoor Air Quality in Delhi}

\section{A. Indoor Air Quality in Low and High SES Households}

In Figure 1, we plot distributions of daytime indoor $\mathrm{PM}_{2.5}$ during the wintertime for low SES households (documented in 2018-19) and high SES households (documented in 2019-20). Indoor $\mathrm{PM}_{2.5}$ levels are extremely high in both samples, with mean concentrations that are 23 and 29 times the WHO safe limit of $10 \mu \mathrm{g} / \mathrm{m}^{3}$, respectively.

In Table 1 we estimate the difference in indoor $\mathrm{PM}_{2.5}$ between high and low SES households, controlling for outdoor $\mathrm{PM}_{2.5}$ and temporal determinants, using the equation:

$$
\log \left(\text { Indoor } \mathrm{PM}_{2.5}\right)_{\mathrm{it}}=\alpha_{0}+\alpha_{1} \operatorname{High~SES}_{\mathrm{i}}+\gamma \log \left(\mathrm{Ambient}_{\mathrm{PM}} \mathrm{P}_{2.5}\right)_{\mathrm{it}}+\delta_{\mathrm{t}}+\omega_{\mathrm{it}}
$$

where the dependent variable is the logged mean indoor $\mathrm{PM}_{2.5}$ for household $i$ during the 15 -minute interval $t$; High $\mathrm{SES}_{\mathrm{i}}$ is a binary variable indicating high SES status; $\log \left(\mathrm{Ambient}^{\mathrm{PM}} \mathrm{M}_{2.5}\right)_{\text {it }}$ is the logged mean $\mathrm{PM}_{2.5}$ concentration from the nearest outdoor government monitor for household $i$ during interval $t ; \delta_{\mathrm{t}}$ are time fixed effects for month-of-year, day-of-month, and hour-of-day; and standard errors are clustered at the sampling level (J.J. cluster for low SES; monitor for high SES). In column $2 b$, our preferred specification, we estimate that high SES households have indoor $\mathrm{PM}_{2.5}$ levels that are 10 percent lower than in low SES households.

\section{B. Differences in Indoor and Outdoor Air Quality}

In Delhi, the primary source of information on air pollution is a network of 36 government monitors deployed across the city by the Central Pollution Control Board, Delhi Pollution Control Committee, and the Indian Meteorological Department. In Figure A6, we plot distributions of the 
difference between the indoor PM2.5 level measured using the IAQMs and the outdoor, ambient $\mathrm{PM}_{2.5}$ levels reported by the nearest government monitor, for both low and high SES households. On average, the indoor $\mathrm{PM}_{2.5}$ level is substantially higher than the corresponding value reported by the nearest government monitor. This pattern is observed in both low and high SES households (mean differences are +114.4 and $+122.3 \mu \mathrm{g} / \mathrm{m}^{3}$, respectively).

Our data does not allow us to precisely explain these differences. There are, however, several possibilities. For instance, considering the significant intra-urban variability in air pollution (e.g., Jerrett et al. 2005), it is possible that the existing set of government monitors do not reflect the hyperlocal, ambient concentrations that are present at the street or neighborhood level. In Figure A7, we use data from the low SES household sample to show that $\mathrm{PM}_{2.5}$ levels reported by the nearest government monitors are exceeded by those captured using the IAQMs outside respondent homes. We do not have comparable outdoor IAQM measures for the high SES sample. Another possibility, which is documented in the existing IAP literature, is that $\mathrm{PM}_{2.5}$ levels are affected by ventilation and cooking habits (e.g., Leung 2015). In Figure A8A, we use data from a high SES household to show how indoor $\mathrm{PM}_{2.5}$ levels tend to spike in the mornings and evenings, when households are most likely to be cooking.

\section{Impacts of IAQMs on Defensive Investments}

Using endline survey data for households in Groups A and B, we estimate the impact of the randomly assigned, free, month-long IAQM user trial on various defensive actions and awareness outcomes. We focus on treatment-on-treated (TOT) results by estimating the equation:

$$
\mathrm{y}_{\mathrm{i}}=\beta_{0}+\beta_{1} \mathrm{KLE}_{\mathrm{i}}+\mathrm{X}_{\mathrm{i}}^{\prime} \Gamma+\delta_{\mathrm{r}}+\epsilon_{\mathrm{i}}
$$

where $y_{i}$ is an outcome of interest for household $i$; KLE $E_{i}$ indicates whether household $i$ experienced the IAQM, and is instrumented with a binary variable indicating treatment status); $X_{\mathrm{i}}$ is a vector 
of household-level characteristics at baseline (which are listed in Appendix A); $\delta_{\mathrm{r}}$ is a survey round fixed effect; and standard errors are clustered at the level of treatment (RWA cluster).

In Table 2, we report the TOT effect for take-up of the subsidized air purifier rental offer and other outcomes. The intervention did not lead to take-up of the air purifier contracts (zero in both control and treatment), an increase in air purifier ownership, or noticeable changes in defensive actions that could potentially reduce IAP exposure. We observe a sizeable, marginally significant effect on an air pollution awareness index, which captures how respondents performed on a basic air pollution-related knowledge quiz) $(0.3 \sigma ; t$-stat $=1.58)$. In addition, we observe a negative effect on recent consumption of air pollution news $(-11.3$ percentage points; $t$-stat $=1.92)$, suggesting that users may have exhibited information avoidance (e.g., Golman, Hagmann, and Loewenstein 2017). These effects, however, do not persist after calculating the false discovery rate (FDR)-adjusted $q$-values (shown in Table A4) corresponding to the estimates in column 2.

In Appendix B, we report on a second experimental comparison in which we evaluate the impact of a visible display screen on indoor $\mathrm{PM}_{2.5}$ levels, as well as survey outcomes, by comparing Group B (standard KLE) and Group C (modified KLE lacking a visible display screen) households. The sample is restricted to high SES households, given that data collection can only occur over a Wi-Fi network. Although we estimate an 8.6 percent decline in indoor $\mathrm{PM}_{2.5}$ concentrations ( $t$-stat $=1.93)$, which we attribute to a 22.8 pp increase in ventilation behavior $(t$-stat $=2.09)$, the results must be interpreted with caution due to imbalance at baseline, heavy attrition, and a small sample size. The general patterns, however, suggest that there may be some households in this setting that will respond to IAQM information by adopting modest changes in inexpensive defensive practices, even if their ownership of air purifiers does not change.

\section{Discussion}


In a related experiment using the same sample of low SES households we study, Baylis et al. (2021) experimentally estimate modest levels of marginal willingness-to-pay for clean air (MWTP) revealed by individual decisions to purchase pollution masks, and show evidence that MWTP may rise with income, and other important dimensions of heterogeneity. This is relatively consistent with the differences in air purifier ownership across socio-economic strata that we observe in our data: high SES households are over 13 times more likely to own air purifiers at baseline, compared to low SES households. However, low take-up of the free IAQM trial and the subsidized air purifier rental offers suggest that, in this sample of medium and high SES households, the demand for IAP information and defensive technologies may be relatively low.

Information gaps about IAP may not be fully addressed by the high-frequency $\mathrm{PM}_{2.5}$ information communicated through an IAQM screen. Other information gaps about the utility of various defensive actions and investments may also exist. Finding complementary ways to connect $\mathrm{PM}_{2.5}$ concentrations to the health consequences of air pollution, for instance, may lead to different outcomes, and deserves further study.

\section{References}

Baylis, Patrick, Michael Greenstone, Kenneth Lee, and Harshil Sahai. 2021. "Pollution Masks and the Demand for Clean Air: Experimental Evidence from Delhi," unpublished manuscript.

Golman, Russell, David Hagmann, and George Loewenstein. 2017. "Information Avoidance." Journal of Economic Literature. 55 (1): 96-135.

Lee, Kenneth, Harshil Sahai, Patrick Baylis, and Michael Greenstone. 2020. "Job Loss and Behavioral Change: The Unprecedented Effects of the India Lockdown in Delhi." COVID Economics. 55: 134-158.

Leung, Dennis Y. C. 2015. "Outdoor-indoor air pollution in urban environment: challenges and opportunity." Frontiers in Environmental Sciences. 2: 69.

Jerrett, Michael, Altaf Arain, Pavlos Kanaroglou, et al. 2005. "A review and evaluation of intraurban air pollution exposure models." Journal of Exposure Science and Environmental Epidemiology. 15: 185-204. 
Figure 1. Indoor $\mathrm{PM}_{2.5}$ levels in low and high SES Delhi households

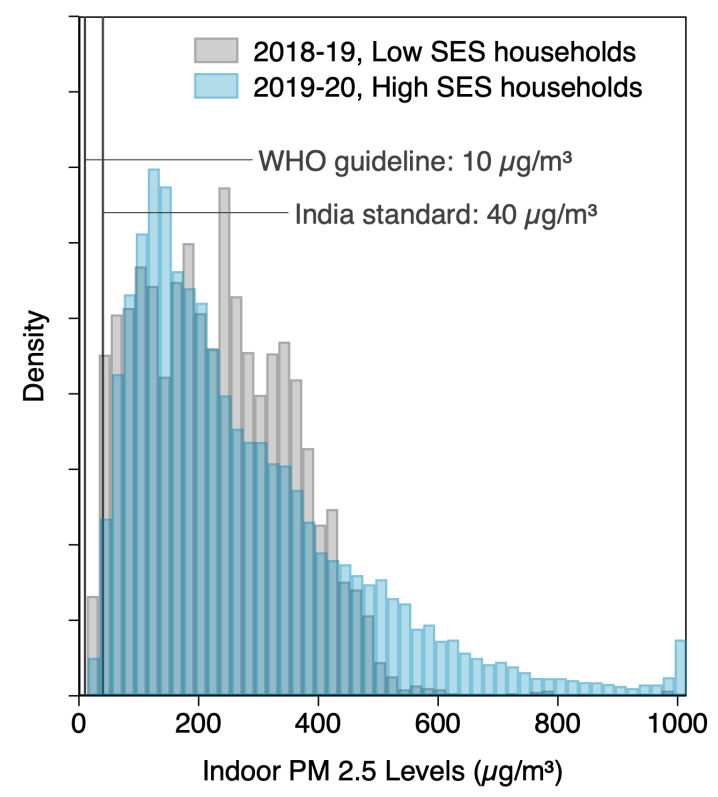

Notes: Indoor $\mathrm{PM}_{2.5}$ measurements recorded between 9AM and 5PM. High SES households are the RWA clusters (Group C) respondents that paired their IAQMs with Wi-Fi. Low SES households are the J.J. clusters respondents. Appendix A includes additional details.

Table 1. Predictors of indoor $\mathrm{PM}_{2.5}$

\begin{tabular}{lcccc}
\hline \hline & \multicolumn{4}{c}{$\mathrm{PM}_{2.5}$ (logged) } \\
\cline { 2 - 5 } & $(1 \mathrm{a})$ & $(1 \mathrm{~b})$ & $(2 \mathrm{a})$ & $(2 \mathrm{~b})$ \\
\hline High SES (=1) & $0.19^{* * *}$ & $-0.09^{* * *}$ & $-0.08^{* *}$ & $-0.10^{* * *}$ \\
& $(0.05)$ & $(0.03)$ & $(0.03)$ & $(0.03)$ \\
Ambient PM 2.5 (logged) & & & $0.80^{* * *}$ & $0.74^{* * *}$ \\
& & & $(0.02)$ & $(0.02)$ \\
\hline Hour, Weekday, Month FEs & No & Yes & No & Yes \\
Observations & 90,295 & 90,295 & 87,937 & 87,937 \\
$\mathrm{R}^{2}$ & 0.00 & 0.22 & 0.64 & 0.68 \\
\hline \hline
\end{tabular}

Notes: Mean indoor $\mathrm{PM}_{2.5}$ is $229 \mu \mathrm{g} / \mathrm{m}^{3}$ and $289 \mu \mathrm{g} / \mathrm{m}^{3}$ for the 3,002 and 55 low and high SES household samples, respectively. Outdoor, ambient $\mathrm{PM}_{2.5}$ measurements are taken from the nearest government monitors. Robust standard errors clustered at the sampling point level are in parantheses. Asterisks indicate coefficient statistical significance level (2-tailed): ${ }^{*} P<0.10 ;{ }^{* *} P<0.05 ;{ }^{* * *} P<0.01$. Appendix A includes additional details. 
Table 2. Impacts of an IAQM user trial on medium and high SES households

\begin{tabular}{|c|c|c|c|}
\hline & $\begin{array}{c}\text { Control } \\
\text { Mean } \\
(1)\end{array}$ & $\begin{array}{l}\text { TOT } \\
\text { Effect } \\
(2)\end{array}$ & $\begin{array}{c}\text { FDR } \\
q \text {-val } \\
(3)\end{array}$ \\
\hline \multicolumn{4}{|l|}{ Panel A: Primary outcome } \\
\hline Accepted subsidized air purifier rental offer $(\%)$ & $\begin{array}{c}0 \\
{[0]}\end{array}$ & $\begin{array}{c}0 \\
(0)\end{array}$ & - \\
\hline \multicolumn{4}{|l|}{ Panel B: Secondary outcomes } \\
\hline Own air purifier (\%) & $\begin{array}{c}5.2 \\
{[22.3]}\end{array}$ & $\begin{array}{l}-0.7 \\
(0.9)\end{array}$ & 0.727 \\
\hline Sealed gaps in home in past month (\%) & $\begin{array}{c}4.7 \\
{[21.2]}\end{array}$ & $\begin{array}{l}-1.6 \\
(3.6)\end{array}$ & 0.777 \\
\hline Closed doors, windows due to outdoor air (\%) & $\begin{array}{c}82.4 \\
{[38.1]}\end{array}$ & $\begin{array}{c}0.8 \\
(4.9)\end{array}$ & 0.866 \\
\hline Lit oil lamp, incense, or candle in past week (\%) & $\begin{array}{c}69.8 \\
{[46.0]}\end{array}$ & $\begin{array}{c}8.3 \\
(7.1)\end{array}$ & 0.635 \\
\hline Air pollution awareness index & $\begin{array}{c}0 \\
{[1]}\end{array}$ & $\begin{array}{l}0.30^{*} \\
(0.19)\end{array}$ & 0.383 \\
\hline Very or extremely concerned (\%) & $\begin{array}{c}61.7 \\
{[48.7]}\end{array}$ & $\begin{array}{c}7.7 \\
(7.7)\end{array}$ & 0.635 \\
\hline Read air pollution news recently (\%) & $\begin{array}{c}55.8 \\
{[49.8]}\end{array}$ & $\begin{array}{c}-11.3^{* *} \\
(5.9)\end{array}$ & 0.383 \\
\hline Used mask in past week (\%) & $\begin{array}{c}15.9 \\
{[36.6]}\end{array}$ & $\begin{array}{l}-2.3 \\
(5.6)\end{array}$ & 0.777 \\
\hline Range of regression sample sizes & & $604-693$ & \\
\hline
\end{tabular}

Notes: Column 1 reports mean values in Group A (no monitor) with standard deviations in brackets. Column 2 reports coefficients from separate TOT (IV) regressions in which the treatment indicator ("Experienced IAQM user trial") is instrumented with a variable indicating whether the household was randomly assigned into Group B (standard KLE). All specifications include respondent and household controls, as well as a survey round fixed effect. Column 3 reports the FDR-adjusted $q$-values associated with the coefficient estimates in column 2. Robust standard errors clustered at the RWA cluster-level in parantheses. Asterisks indicate coefficient statistical significance level (2-tailed): ${ }^{*} P<0.10 ;{ }^{* *} P<0.05$; ${ }^{* *} P<0.01$. Appendix A includes additional details. 